Ken Yamamoto · Eiichi Ishii · Hisanori Horiuchi

Ikuyo Ueda - Shouichi Ohga $\cdot$ Masanori Nishi

Yoshiyasu Ogata · Masafumi Zaitsu · Akira Morimoto

Toshiro Hara · Shinsaku Imashuku

Takehiko Sasazuki · Masaki Yasukawa

\title{
Mutations of syntaxin 11 and SNAP23 genes as causes of familial hemophagocytic lymphohistiocytosis were not found in Japanese people
}

Received: 29 June 2005 / Accepted: 29 July 2005 / Published online: 23 September 2005

(C) The Japan Society of Human Genetics and Springer-Verlag 2005

\begin{abstract}
Although mutations of perforin, MUNC13-4 and syntaxin 11 genes have been found in children with familial hemophagocytic lymphohistiocytosis (FHL), the incidence of each genetic subtype varies in different ethnic groups. We evaluated mutations of syntaxin 11 and $S N A P 23$ genes in 30 Japanese FHL patients. The patients had no mutations and $10 \%$ had one polymorphism $(146 \mathrm{G}>\mathrm{A})$ of syntaxin 11, while no mutation of SNAP23 was observed. Our results indicate that
\end{abstract}

K. Yamamoto

Department of Molecular Genetics,

Medical Institute of Bioregulation, Kyushu University, Fukuoka, Japan

E. Ishii $(\square) \cdot$ M. Nishi $\cdot$ Y. Ogata $\cdot$ M. Zaitsu

Department of Pediatrics, Faculty of Medicine, Saga University, 5-1-1 Nabeshima, Saga 849-8501, Japan

E-mail: ishiei@med.saga-u.ac.jp

Tel: + 81-952-34-2314

Fax: + 81-952-34-2064

H. Horiuchi

Department of Cardiovascular Medicine,

Graduate School of Medicine, Kyoto University, Kyoto, Japan

I. Ueda $\cdot$ A. Morimoto

Department of Pediatrics,

Kyoto Prefectural University of Medicine, Kyoto, Japan

S. Ohga $\cdot$ T. Hara

Department of Pediatrics, Graduate School of Medical Sciences,

Kyushu University, Fukuoka, Japan

S. Imashuku

Division of Pediatrics, Takasago Seibu Hospital, Takasago, Japan

T. Sasazuki

Research Institute, International Medical Center of Japan,

Tokyo, Japan

M. Yasukawa

First Department of Internal Medicine, Ehime University, Ehime, Japan aberrations in the SNARE system may not cause FHL in Japanese families.

Keywords Infant - Familial hemophagocytic

lymphohistiocytosis · Syntaxin $11 \cdot$ SNAP23 .

Cytotoxicity $\cdot$ Japan

\section{Introduction}

Familial hemophagocytic lymphohistiocytosis (FHL) is a rare autosomal recessive inherited disorder that usually arises during infancy (Henter et al 1991). The major pathogenesis of FHL is associated with defects in natural killer (NK) cells and cytotoxic $\mathrm{T}$ lymphocytes (CTLs); most FHL patients have low or deficient NK cell and CTL activity due to the impairment of lytic granule secretion used for elimination of target cells (Schneider et al 2002; Yanai et al 2003). Thus, identifying the molecular genetic defects that underlie FHL has been a major concern.

In recent years, mutations of the perforin PRF1, MIM170280) and MUNC13-4 (MIM608897) genes have been found in FHL patients (Stepp et al 1999; Feldmann et al 2003). Both FHL-causing perforin and MUNC13-4 are involved in the cytotoxic pathway of NK cells and CTLs (Stinchcombe et al 2004). Based on our several reports, PRF1 or MUNC13-4 gene mutations cause about $20-30 \%$ or $20-25 \%$ of all registered FHL2 or FHL3 cases in Japan (Suga et al 2002; Ueda et al 2003; Yamamoto et al 2004). Recently, mutation of syntaxin 11 (STX11) located on chromosome 6q24 was identified as a cause of FHL4 in Kurdish/Turkish families; homozygous deletion in four families and homozygous nonsense mutation in two families resulted in a premature stop codon, and complete loss of syntaxin 11 (zur Stadt et al 2005). However, the mutation of this gene was not found in any FHL pa- 
tient of non-Kurdish/Turkish origin (zur Stadt et al 2005), suggesting that STX11 mutation can be seen in specific ethnic groups.

Syntaxin 11 is a soluble $N$-ethylmaleimide-sensitive factor attachment protein receptor present on target membranes ( $t$-SNAREs) (Tang et al 1998; Chen et al 2001). Upon comparison with other members of $t$ SNAREs such as SNAP25 and SNAP29, SNAP23 was identified as being a binding partner with syntaxin 11 in the secretory process (Valdez et al 1999). These facts prompted us to evaluate mutations of STX11 and $S N A P 23$ genes in Japanese FHL patients.

\section{Subjects and methods}

Japanese patients who met the diagnostic criteria for hemophagocytic lymphohistiocytosis (HLH), which includes prolonged intermittent fever and splenomegaly as clinical findings, and cytopenia, hypertriglyceridemia, hypofibrinogenemia and hemophagocytosis as laboratory findings (Henter et al 1991), were eligible for the study. Among them, a total of 69 patients were suspected of having FHL for the following reasons; young age at onset, central nervous system (CNS) involvement, NK cell deficiency or positive family history (Ishii et al 1998). Documented informed consent was obtained from the parents of each patient according to the Declaration of Helsinki. The study was also approved by the Institutional Review Boards at Kyushu University and Saga University, Japan. As described in our recent report (Ishii et al 2005), all cases were screened for the PRF1 gene, 12 of which were classified as FHL2. Thirty-eight of the remaining 57 cases were tested for MUNC13-4 gene mutations, eight of which were diagnosed as FHL3. Subsequently, the remaining 30 patients with neither PRF1 nor MUNC13-4 mutations were tentatively classified as non-FHL2/3, and eligible for this study. The gender distribution of this group was 17 males and 13 females. The median age at onset of disease was 11 months (from day 0 to 96 months); disease onset occurred at less than 12 months of age in 16 patients. Family history was positive in 11 patients. CNS involvement, defined as pleocytosis in the cerebrospinal fluid or abnormal findings by computed tomography, was detected in 12 patients at presentation. NK cell activity was deficient ( $4 \%$ or less) in nine, moderately decreased $(5-17 \%)$ in 12 , and normal $(18-40 \%)$ in two patients. Most of the patients initially received combination chemotherapy including steroid, etoposide and cyclosporine A with or without other cytotoxic drugs, followed by hematopoietic stem cell transplantation (HSCT). All of the ten patients who underwent allogeneic HSCT are alive. Among the patients who did not receive HSCT, seven died of progressive disease or various complications.

For the detection of STX11 and SNAP23 mutations, genomic DNA was isolated from the peripheral blood mononuclear cells (PBMC) of each patient. Genomic DNA (10 ng) was subjected to PCR using primers listed in Table 1. Those primer sets amplify two or eight exons including $5^{\prime}$ UTR and the coding regions with exonintron boundaries of the STX11 or SNAP23 gene, respectively. Each reaction contained $2.5 \mathrm{mM} \mathrm{MgCl}_{2}$, $200 \mathrm{nM}$ dNTPs, $250 \mathrm{nM}$ of both forward and reverse primers and 0.5 unit of rTaq DNA polymerase (Takara Bio, Otsu, Japan). Amplification was carried out as follows: 35 cycles of $96^{\circ} \mathrm{C} / 30 \mathrm{~s}, 55^{\circ} \mathrm{C} / 30 \mathrm{~s}$ and $72{ }^{\circ} \mathrm{C} /$ $30 \mathrm{~s}$, and final extension at $72{ }^{\circ} \mathrm{C} / 7 \mathrm{~min}$. PCR products were treated with ExoSAP-IT (Amercham Pharmacia Biotech, Uppasala, Sweden) by incubating at $37^{\circ} \mathrm{C}$ for 15 min to inactivate free primers and dNTPs, and then purified using Magnesil (Promega Corporation, Madison, WI, USA). Sequencing of PCR products was performed with an ABI 3730 Genetic Analyzer (Applied Biosystems, Foster City, San Francisco, CA, USA). Forward and reverse primers were used to obtain both strand sequences. Sample sequences were aligned to reference genomic sequences obtained from the UCSC Genome Bioinformatics Site (http://genome.ucsc.edu/ index.html) with the ClastalW program in order to identify nucleotide changes.

Table 1 Primer sets used to detect the genetic mutations of STX11 and SNAP23 in FHL patients

\begin{tabular}{lll}
\hline & Forward primer & Reverse primer \\
\hline STX11 & & \\
STX11-1 & AGCTGTGGTCGCTTCCTAAG & AAAATCCCACCTAAGGCACA \\
STX11-2 & CCCACACCGAGGAATACAAA & CTTGGCGATGGAGTTGGT \\
STX11-3 & GAGACATCCGGGACATTCAG & CAGCAAGTTCTCGGAAAACAC \\
STX11-4 & CCATGCACGACTACAACCAG & GCTCCATGGTCTGGGATG \\
SNAP23 & & \\
SNAP23-1 & GACATTTAAACCCCCAGCAC & CGTCCCACTCCTGGAGACT \\
SNAP23-2 & TTTCCTGATAAGTCCTAAATTCCA & AAGGCTCTCTCACTCCTCCA \\
SNAP23-3 & GCCTGAGCTGGCAATTTTA & CCAGCCAGAAGTATTAGGATCG \\
SNAP23-4 & TCCTCTGAGAGCTTGGGTTT & TGATAGGGCTTTTGGCATC \\
SNAP23-5 & CTCACTGTGGGGAGTTATTGG & GTGGGAGAAGGTGGGGTACT \\
SNAP23-6 & TTGGTATCTCCCTACACTCCTGA & TTTTATTTGTTTATTAGGGGTCATGT \\
SNAP23-8 & CTTGCTGAGAGTCATTTTGGTT & GGGTGTCTCATTTTCTTGTGG \\
\hline
\end{tabular}


Table 2 Results from mutational analysis of $S T X 11$ and $S N A P 23$ in Japanese nonFHL-2/3 patients

${ }^{\mathrm{a}} 146 \mathrm{G}>\mathrm{A}$, heterozygosity
STX11

SNAP23

Exon 1

Exon 2

Number of mutations

Number of polymorphisms

\section{$0 / 30$}

$0 / 30$
$0 / 30$

$3 / 30(10.0 \%)^{\mathrm{a}}$
$0 / 30$

$0 / 30$

\section{Results}

The results are shown in Table 2. FHL patients had no mutations, and $10 \%$ had one non-synonymous cSNP (146G $>$ A, rs17073498) of $S T X 11$, the minor allele, with a reported frequency of 0.035 in the dbSNP build 124 database (NCBI, http://www.ncbi.nlm.nih.gov/). Neither mutations nor polymorphisms of $S N A P 23$ were observed in FHL patients. Our results indicate that aberrations in the SNARE system (involving $S T X 11$ and $S N A P 23)$ do not cause FHL in Japanese patients. In addition to the current 30 non-FHL2/3 Japanese patients, we also studied three additional cases from other Asian countries, but no mutations were found in these genes either (data not shown).

\section{Discussion}

The molecular mechanism of vesicle transport has been clarified in recent years. The final step of vesicle transport is mediated by a bridge between a vesicle and target membrane through ternary complex formation between vesicle-soluble $\quad N$-ethylmaleimide-sensitive factor attachment protein receptors ( $v$-SNARE) such as a VAMP and $t$-SNARE such as a syntaxin 11 or a member of SNAP23/25/29 (Chen et al 2001). Syntaxin 11, in association with SNAP23, localizes to the endosome and trans-Golgi network (Valdez et al 1999); however, the precise cell-biological functions of syntaxin 11 are currently poorly understood. Although SNARE proteinmediated lytic granule exocytosis in NK cells and CTL has not yet been identified, syntaxin 11 and SNAP23 might mediate the process. Alternatively, since lytic granules are classified as secretory lysosomes, syntaxin 11 might be involved in the regulation of lytic granule biogenesis rather than granule secretion (Stinchcombe et al 2004).

The incidence of each genetic subtype of FHL varies among different ethnic groups. In Japan, the incidence of FHL3 is nearly equal to or slightly higher than that of FHL2, while the incidence of either FHL2 or FHL3 is reportedly about $25 \%$ in Germany (Molleran Lee et al 2004; zur Stadt et al 2004). Many cases of FHL2 have been described in African-Americans, whites, Hispanics and some Asians, but so far no FHL3 cases have been reported in the US. In Turkey, FHL2 seems to be the major type of FHL, while the $S T X 11$ mutation also appears to be present in this ethnic group. In contrast, this mutation was not detected in any FHL patient of
non-Kurdish/Turkish origin (zur Stadt et al 2005). In the current study, mutations of STX11 and SNAP23 genes were not detected in FHL patients from Japan and other Asian countries. This suggests that the $S T X 11$ mutation of FHL is limited to specific ethnic groups. Thus, identification of the new genetic defects in $\sim 50 \%$ of the FHL patients in Japan remains a major concern.

\section{Electronic database information}

Accession numbers and URLs for data presented herein are as follows.

GenBank accession number. cDNA sequences of STX11 and SNAP23, NM_003764 and NM_003825.

The Genome Browser of UCSC Genome Bioinformatics, http://genome.ucsc.edu/index.html (for genome sequences of $S T X 11$ and $S N A P 23)$.

Whitehead Institute for Biomedical Research, http:// www.broad.mit.edu/ (for picking primers to amplify STX11 and SNAP23 genes).

Acknowledgements We thank all patients and their family members who participated in this study. We also wish to thank all members of the Japan FHL study group. This work was supported by a Grant-in-Aid for Scientific Research and a Grant from the 21st COE Program from the Ministry of Education, Culture, Sports, Science and Technology, Japan.

\section{References}

Chen YA, Scheller RH (2001) SNARE-mediated membrane fusion. Nat Rev Mol Cell Biol 2:98-106

Feldmann J, Callebaut I, Raposo G et al (2003) Munc13-4 is essential for cytolytic granules fusion and is mutated in a form of familial hemophagocytic lymphohistiocytosis (FHL3). Cell 115:461-473

Henter J, Elinder G, Ost A (1991) Diagnostic guidelines for hemophagocytic lymphohistiocytosis. Sem Oncol 18:29-33

Ishii E, Ohga S, Tanimura M et al (1998) Clinical and epidemiologic studies of familial hemophagocytic lymphohistiocytosis in Japan. Med Pediatr Oncol 30:276-283

Ishii E, Ueda I, Shirakawa R et al (2005) Genetic subtypes of familial hemophagocytic lymphohistiocytosis: correlations with clinical features and cytotoxic T lymphocyte/natural killer cell functions. Blood 105:3442-3448

Molleran Lee S, Villanueva J, Sumegi J et al (2004) Characterization of diverse PRF1 mutations leading to decreased natural killer cell activity in North American families with haemophagocytic lymphohistiocytosis. J Med Genet 41:137-144

Schneider EM, Lorenz I, Muller-Rosenberger M, Steinbach G, Kron M, Janka-Schwarb GE (2002) Hemophagocytic lymphohistiocytosis is associated with deficiencies of cellular cytosis but normal expression of transcripts relevant to killer-cell-induced apoptosis. Blood 100:2891-2898 
Stepp SE, Dufourcq-Lagelouse R, Le Deist F et al (1999) Perforin gene defects in familial hemophagocytic lymphohistiocytosis. Science 286:1957-1959

Stinchcombe J, Bossi G, Griffiths GM (2004) Linking albinism and immunity: the secrets of secretory lysosomes. Science 305:55-59

Suga N, Takada H, Ohga S et al (2002) Perforin defects of primary haemophagocytic lymphohistiocytosis in Japan. Br J Haematol 116:346-349

Tang BL, Low DY, Hong W (1998) Syntaxin 11: a member of the syntaxin family without a carboxyl terminal transmembrane domain. Biochem Biophys Res Commun 245:627-632

Ueda I, Ishii E, Morimoto A, Ohga S, Sako M, Imashuku S (2005) Correlation between phenotypic heterogeneity and gene mutational characteristics in familial hemophagocytic lymphohistiocytosis (FHL). Pediatr Blood Cancer (in press)

Ueda I, Morimoto A, Inaba T et al (2003) Characteristic perforin gene mutations of haemophagocytic lymphohistiocytosis patients in Japan. Br J Haematol 121:503-510
Valdez AC, Cabaniols JP, Brown MJ, Roche PA (1999) Syntaxin 11 is associated with SNAP-23 on late endosomes and the transGolgi network. J Cell Sci 112:845-854

Yamamoto K, Ishii E, Sako M, et al (2004) Identification of novel MUNC13-4 mutations in familial hemophagocytic lymphohistiocytosis and functional analysis of MUNC13-4-deficient cytotoxic T lymphocytes. J Med Genet 41:763-767

Yanai F, Ishii E, Kojima K et al (2003) Essential roles of perforin in antigen-specific cytotoxicity mediated by human $\mathrm{CD}^{+}{ }^{+} \mathrm{T}$ lymphocytes: analysis using the combination of hereditary perforin-deficient effector cells and Fas-deficient target cells. J Immunol 170:2205-2213

zur Stadt U, Beutel K, Oyen F et al (2004) UNC13D and PRF1 mutations in childhood patients with hemophagocytic lymphohistiocytosis. Blood 104:375a-376a (Abstract)

zur Stadt U, Schmidt S, Kasper B et al (2005) Linkage of familial hemophagocytic lymphohistiocytosis (FHL) type-4 to chromosome 6q24 and identification of mutations in syntaxin 11 . Hum Mol Genet 14:827-834 